\title{
Influencia Del Comité De Auditoría En El Desempeño Financiero Organizacional, En Empresas Mexicanas Cotizadas (2010-2014) ${ }^{22}$
}

\author{
Dr. Sergio Demetrio Polo Jiménez \\ Dr. Eleazar Villegas González \\ Mtra. Martha Jiménez Alvarado \\ C. Daniela Martín Benítez
}

Universidad Autónoma del Estado de Hidalgo, Mexico

Doi:10.19044/esj.2018.v14n19p135 URL:http://dx.doi.org/10.19044/esj.2018.v14n19p135

\begin{abstract}
The purpose of this paper is to know the level of compliance with corporate practices related to the audit function, contained in the Code of Best Corporate Practices, issued by the National Banking and Securities Commission and influence on organizational financial performance (specifically on financial profitability), for this, a sample of 405 questionnaires of best corporate practices, belonging to 125 non-financial companies, are listed on the Mexican Stock Exchange during the period, 2010-2014. The results of the application of the linear regression model, could allow us to affirm that the Audit Function (INDEX_FA), increases the financial profitability of the organizations, which is a great success on the part of the Mexican regulators.
\end{abstract}

Keywords: Corporate Governance, Financial Performance, Audit Function, Profitability

\section{Resumen}

El propósito del presente trabajo, se orienta en conocer el nivel de cumplimiento de las prácticas societarias relativas a la función de auditoria, contenidas en el Código de Mejores Prácticas Corporativas (CMPC), emitido por la Comisión Nacional Bancaria y de Valores (CNBV) y su influencia en el desempeño financiero organizacional (específicamente en la rentabilidad financiera), para ello, se analiza una muestra formada por 405 cuestionarios de mejores prácticas corporativas, pertenecientes a 125 empresas no

\footnotetext{
${ }^{22}$ Se agradece la colaboración en la realización del presente trabajo de investigación del alumno de la Licenciatura en Contaduría de la Universidad Autónoma del Estado de Hidalgo, Marco Antonio Aparicio Ramírez.
} 
financieras, que cotizan en la Bolsa Mexicana de Valores (BMV) durante el periodo, 2010-2014. Los resultados de la aplicación del modelo de regresión lineal, nos podrían permitir afirmar que la Función de Auditoria (INDEX_FA), aumenta la rentabilidad financiera de las organizaciones, lo que representa, un gran acierto por parte de los organismos reguladores mexicanos.

Keywords: Gobierno Corporativo, Desempeño Financiero, Función de Auditoria, Rentabilidad

\section{INTRODUCCIÓN}

Las organizaciones, revisten una importancia vital en cualquier sociedad, con su apoyo se pueden alcanzar progresos económicos, generación de riqueza y son fuentes constantes de empleo además de producir bienes y servicios necesarios; por lo que su permanencia es de interés no solo para los gobiernos sino también para sus propietarios y demás personas que dependen de ellas. En México el Consejo Coordinador Empresarial, estableció en 1999 el Comité de Mejores Prácticas Corporativas, quien a su vez emitió por primera ocasión en 2006 el "Código de Mejores Prácticas Corporativas (CMPC)", en donde fueron establecidas diversas recomendaciones que el consejo de administración debe tomar en consideración para ayudar a las organizaciones a tener una mayor transparencia en las operaciones que realizan y que son reflejadas a través de la emisión de la información contable, de acuerdo a las normas establecidas para ese efecto y la regulación legal aplicable. Actualmente, existe una segunda versión publicada en Abril de 2010, que toma en cuenta las necesidades y características de las empresas mexicanas, siempre buscando la forma de ayudarles a ser más competitivas y perdurables a través del tiempo.

El CMPC, contiene lo relativo a la Función de Auditoría (FA) y recomienda la existencia de un órgano intermedio que apoye las actividades del Consejo de Administración (CA), en la función de revisión, buscando un equilibrio entre la Auditoría Interna y Externa, para que los resultados de su trabajo sean emitidos de forma objetiva y revelada, con total apego a las disposiciones aplicables, adicionalmente, también se recomienda que el control interno existente sea validado constantemente y que estas funciones sean llevadas a cabo y que en el caso de necesitar alguna corrección, se haga la propuesta correspondiente por el Comité hacia el Consejo de Administración, para que sea este, quien tome las decisiones correspondientes y materialice los cambios más adecuados. Las prácticas de Gobierno Corporativo (GC), son consideradas como herramientas que contribuyen a mejorar la integración y el funcionamiento del Consejo de Administración y sus órganos intermedios de apoyo, uno de ellos, es el Comité de Auditoría, el cual entre sus principales funciones, tiene la misión de asegurar que la 
Auditoría Interna y la Auditoría Externa se realicen con objetividad e independencia, procurando que la información que se produzca sea siempre suficiente y oportuna, reflejando razonablemente la situación financiera de la sociedad. La implantación y seguimiento de dichas prácticas dentro de las organizaciones, han coadyuvado a incrementar la competitividad en los diferentes mercados internacionales, así como también garantizar la protección del capital de los inversores y las fuentes de empleo, a través de la aplicación de la mejores prácticas corporativas, se busca apoyar a las sociedades en aspectos tan importantes, como la transparencia de su operación y la revelación de su información; lo cual generaría condiciones económicas favorables, permanencia estable en los mercados, beneficios para sus accionistas y demás interesados.

Dado lo anterior, el trabajo de investigación está orientado en conocer, por un lado, el grado de cumplimiento de los mecanismos de gobierno corporativo relativos a la Función de Auditoria por parte de las empresas de la muestra y por el otro, si estas prácticas societarias, ejercen influencia significativa en el performance organizacional, este último medido a través de la rentabilidad financiera, como lo han evidenciado diversos trabajos de investigación alrededor del mundo. Para alcanzar los objetivos propuestos, se estructura el estudio de la siguiente manera: tras la introducción, se presenta la revisión de la literatura previa, se continúa con el diseño y metodología de la investigación, describiendo los objetivos, la muestra y definiendo las variables objeto de estudio; acto seguido se dan a conocer los resultados de los análisis descriptivos e inferenciales y terminamos con la exposición de las principales conclusiones y la bibliografía utilizada.

\section{REVISIÓN DE LA LITERATURA}

Son abundantes los estudios, que tratan de conocer la relación que existe entre los mecanismos del Gobierno Corporativo y determinadas características del desempeño organizacional alrededor del mundo, en la opinión de Stefanescu (2011), quien con el propósito de verificar información que garantice el apego a los Códigos de Gobierno Corporativo, analiza 27 estados miembros de la Unión Europea, sus resultados le permiten concluir que el cumplimiento a las disposiciones contenidas en estos códigos, influye determinantemente en la situación económica de los estados sujetos a análisis. Por la importancia de los trabajos, destacan entre otros, los realizados por:

Benavides \& Mongrut (2010), quienes analizan una muestra formada por 199 empresas colombianas, con el objetivo de estudiar los efectos sobre el desempeño contable y sobre las decisiones financieras de estas empresas, después de implementar su código de gobierno, sus resultados muestran, que el retorno sobre los activos luego de aplicar el código, mejora en más del 1\%, el efecto es amplificado por la calidad del código. Dos años más tarde, el 
estudio de Puspitaninggrum, (2012) analiza a 420 empresas listadas en el mercado financiero de Indonesia, sus resultados muestran que los mecanismos de Gobierno Corporativo (Estructura de Propiedad, Comisarios Independientes y el Comité de Auditoría), afectan el nivel de revelación en la emisión voluntaria de Información Financiera a través de Internet.

De la misma manera, el trabajo presentado por Chen \& Chen (2012), quienes a través de un análisis de regresión lineal, sus resultados les permiten concluir que las empresas con mecanismos internos o externos de Gobierno Corporativo más efectivos, experimentan asignaciones de inversión más eficientes, tanto en la empresa como en sus segmentos. La eficiencia del proceso de asignación de la inversión es mejor para las empresas diversificadas, con alta independencia de la junta de accionistas, alta identificación institucional, alta titularidad del director externo, mayor remuneración de la gerencia basada en la equidad, mayor calidad de la auditoría y fuertes derechos de los accionistas, lo que significa, que las consideraciones del Gobierno Corporativo son importantes para evaluar la relación entre la eficiencia de la inversión y el valor de la empresa. Por su parte, Jiraporn \& Kim (2012), con el propósito de conocer los efectos de la adecuación de Gobierno Corporativo en el desempeño de las empresas manufactureras coreanas, analizan una muestra formada por 398 compañías.

A través de un modelo de regresión lineal, sus resultados muestran que la relación entre la adecuación de Gobierno y el desempeño corporativo es estadísticamente significativa.

En México, Martínez (2011), evaluó el impacto generado entre la aplicación de la ley del Código del Buen Gobierno, los resultados de la empresa y el desempeño de su junta de Consejo, en una muestra de empresas no financieras que han cotizado en la Bolsa Mexicana de Valores (BMV) en el decenio comprendido desde 1999 hasta 2009, sus resultados le permitieron conocer la existencia de una relación significativa entre los resultados financieros de la empresa y determinadas dimensiones del Gobierno Corporativo. En el mismo sentido, el trabajo desarrollado por Jiménez, Gallardo, \& Alvarado (2013), analizó una muestra de empresas no financieras que cotizan de nueva cuenta en el mercado mexicano en el periodo 2005-2010, con el propósito de investigar si el cumplimiento de las mejores prácticas corporativas tienden a aumentar la competitividad de las empresas. A través de un modelo de regresión lineal, determina que las empresas que no siguen las recomendaciones del Código de Mejores Prácticas Corporativas en razón a la independencia del Consejo de Administración, al tamaño del Comité de Auditoría, así como la dualidad presidente/director general, reflejan menos rentabilidad empresarial. Algunos trabajos, que se han centrado en identificar la influencia de determinadas dimensiones específicas de los mecanismos del Gobierno Corporativo en el desempeño organizacional son los realizados por 
(Lakshan \& Wjekoon, 2012; Cueto, 2013; Feras, 2013; Lim, 2013; Bota, 2013;; Chris, Theodoros, \& Vasilios, 2014; Latief, Hassan, \& Ahmed, 2014; Abbadi, Hijazi, \& Rahahleh, 2016). Así mismo, Prommin, Jumreornvong, \& Jiraporn (2014), con la intención de conocer el impacto de los mecanismos del Gobierno Corporativo en la liquidez empresarial, analizan una muestra de 100 empresas que cotizan en la Bolsa de Valores de Tailandia, sus resultados muestran, una relación significativa entre las prácticas societarias y la liquidez empresarial.

Otro estudio que resalta la importancia de las prácticas societarias, es el desarrollado por Lagos (2014), que tiene como propósito, estudiar la relación entre la aplicación de prácticas del Gobierno Corporativo, incluidas en el Código de Mejores Prácticas Corporativas y el costo de capital, proveniente de la deuda de empresas listadas en la Bolsa de Valores de Colombia. A través de un modelo de regresión lineal, sus resultados muestran que el nivel de Gobierno Corporativo comienza a ser relevante en el momento de decidir sobre una inversión, debido a que las prácticas corporativas de una empresa, reflejan de cierto modo algunos elementos a considerar en el nivel de riesgo; fundamentalmente permite a los inversionistas contar con mejores niveles de confianza en sus inversiones, esto permite deducir que la aplicación de prácticas de Gobierno Corporativo comienza a ser reconocida en el mercado de valores colombiano, como un indicador que refleja el desempeño de las empresas y no como un simple requisito para la emisión de títulos valores. Así mismo, Javaid \& Saboor (2015), analizan una muestra formada por 58 empresas manufactureras cotizadas en el mercado financiero de Karachi en el periodo de 2009-2013, con el propósito de conocer empíricamente la relación que guarda el Gobierno Corporativo y el performance organizacional en términos financieros, así como el desempeño del mercado medido por retorno de los activos y recursos propios. A través de la aplicación de un modelo de regresión lineal, sus resultados les permiten comprobar la existencia de una relación significativa de tipo positivo entre el índice de GC y el performance de las empresas analizadas, lo que significa que, a mayor GC aplicado, mayor es el comportamiento financiero, el retorno de los activos y los recursos propios. Por último, el estudio realizado por Acosta, Barreda, Diaz, \& Visso (2016), se orienta en comprobar cómo las prácticas de Buen Gobierno Corporativo, influyen en el valor de las empresas. Para ello, se analiza el impacto de las prácticas societarias en los estados financieros en el período 2011-2014, de dos empresas del sector minero que cotizan en la Bolsa de Valores de Lima. Sus resultados no encuentran indicios estadísticos que ayuden a concluir o afirmar que la adopción de una buena gestión de Gobierno Corporativo representada en la adopción de las Prácticas de Gobierno Corporativo redunda en el incremento del valor de las empresas que las desarrollan. 


\section{DISEÑO Y METODOLOGÍA OBJETIVOS}

El propósito del presente trabajo, es conocer la influencia que mantienen los mecanismos de Gobierno Corporativo relativos a la Función de Auditoría (contenidos, en el Código de Mejores Prácticas Corporativas) y determinadas características empresariales en el performance organizacional, específicamente en la rentabilidad financiera de las organizaciones no financieras, listadas en la Bolsa Mexicana de Valores en el periodo 2011-2014. Específicamente se pretende:

- Conocer si las prácticas societarias relativas a la función de Auditoría, aumentan la rentabilidad económica de las organizaciones que cotizan en la BMV.

- Identificar la relación entre el año del informe y la rentabilidad financiera de las empresas que cotizan en la BMV del periodo de estudio

- Conocer si el sector en el que se ubican las empresas de acuerdo a la clasificación de la BMV, influye en la rentabilidad financiera de las mismas.

- Analizar si el tamaño medido a través de sus activos al final de cada ejercicio del periodo, mantiene una relación con la rentabilidad financiera de las empresas de la muestra.

- Verificar si la región en la que se ubican las empresas de la muestra mantiene una relación significativa con la rentabilidad financiera de las empresas de la muestra.

\section{DESCRIPCIÓN DE LA MUESTRA}

Con la finalidad de alcanzar los objetivos establecidos, se analiza una muestra formada por 405 cuestionarios de mejores prácticas corporativas, pertenecientes a 125 empresas de tipo no financiero que cotizan en la Bolsa Mexicana de Valores durante el periodo de 2010 a 2014, la elección de dichas empresas es justificada ya que estas están obligadas a revelar su información financiera, así como el apego al código de mejores prácticas corporativas, lo cual puede ser considerado como información pública que puede consultarse por cualquier persona interesada. El detalle de la muestra puede apreciarse en la Tabla 1: 
Tabla 1: Detalle de la muestra

\begin{tabular}{|l|c|c|c|c|c|c|}
\hline \multicolumn{1}{|c|}{ Sector/Año } & $\mathbf{2 0 1 0}$ & $\mathbf{2 0 1 1}$ & $\mathbf{2 0 1 2}$ & $\mathbf{2 0 1 3}$ & $\mathbf{2 0 1 4}$ & Total \\
\hline Materiales & 11 & 11 & 11 & 17 & 20 & 70 \\
\hline Industrial & 21 & 21 & 20 & 27 & 36 & 125 \\
\hline Servicios y bienes de consumo básico & 13 & 13 & 13 & 14 & 24 & 77 \\
\hline Salud & 3 & 3 & 3 & 4 & 4 & 17 \\
\hline Productos de consumo frecuente & 14 & 14 & 14 & 18 & 19 & 79 \\
\hline Telecomunicaciones & 6 & 6 & 6 & 8 & 11 & 37 \\
\hline Totales & $\mathbf{6 8}$ & $\mathbf{6 8}$ & $\mathbf{6 7}$ & $\mathbf{8 8}$ & $\mathbf{1 1 4}$ & $\mathbf{4 0 5}$ \\
\hline
\end{tabular}

Fuente: Elaboración Propia

Concretamente, para cada empresa disponemos de la siguiente información:

- Código de mejores prácticas corporativas por los años 2010-2014

- Tamaño de la empresa (medido por el total de activos al 31 de diciembre de cada año).

- Informes anuales 2010-2014

- Rentabilidad Financiera

- Sector (tomado de la clasificación que realiza la BMV)

\section{HIPÓTESIS}

Para alcanzar los objetivos planteados y atendiendo lo establecido por la literatura previa, como es el caso del trabajo desarrollado por Jiménez, Gallardo, \& Alvarado (2013), en relación con la rentabilidad se establecen las siguientes hipótesis:

- $\mathrm{H}_{1}$ : El órgano intermedio que desempeña la función de auditoría influye significativamente en la rentabilidad de la organización.

- $\mathrm{H}_{2}$ : El año del informe influye en la rentabilidad financiera.

- $\mathrm{H}_{3}$ : El sector en el que se ubican las empresas, influye en la rentabilidad de la empresa.

- H4: La región en la que se ubican las empresas mantiene influencia significa en la rentabilidad de la empresa.

- H5: El tamaño de las empresas, es un factor de influencia de la rentabilidad financiera

\section{VARIABLES DEPENDIENTE E INDEPENDIENTES}

A continuación, se presenta la relación de variables (dependientes e independientes) que son sujetas de estudio y consideradas predictivas del performance organizacional, (Tabla 2): 
Tabla 2: Descripción de variables dependientes e independientes

\begin{tabular}{|l|l|l|l|}
\hline \multicolumn{1}{|c|}{ Siglas } & \multicolumn{1}{|c|}{ Nombre } & Tipo de Variable & \multicolumn{1}{c|}{ Cálculo } \\
\hline RF & Rentabilidad Financiera & Dependiente & $\begin{array}{l}\text { RF= RN/CC } \\
\text { RF=Resultado neto / } \\
\text { Capital Contable }\end{array}$ \\
\hline Se & Sector de Pertenencia & Independiente & $\begin{array}{l}\text { De acuerdo a la } \\
\text { clasificación dada por } \\
\text { BMV en 6 sectores }\end{array}$ \\
\hline A & Año de Informe Anual & Independiente & $\begin{array}{l}\text { Año que corresponde el } \\
\text { código de mejores } \\
\text { prácticas corporativas }\end{array}$ \\
\hline T & $\begin{array}{l}\text { Región del país } \\
\text { encuentra la Empresa }\end{array}$ & Independiente & $\begin{array}{l}\text { Clasificada en 3 valores: } \\
\text { 1.-Norte, 2.- Centro 3.-Sur }\end{array}$ \\
\hline INDEX_FA & $\begin{array}{l}\text { Índice de la función de } \\
\text { Auditoria }\end{array}$ & Independiente & $\begin{array}{l}\text { Calculada en función del } \\
\text { tamaño de sus activos al 31 } \\
\text { de Diciembre de cada año }\end{array}$ \\
\hline
\end{tabular}

Fuente: Elaboración Propia

\section{ANÁLISIS DESCRIPTIVOS}

Con el propósito, de dar a conocer los resultados del análisis descriptivo de los constructos relativos a la Función de Auditoria, contenidos en el cuestionario de mejores prácticas societarias, emitido por el Consejo Coordinador Empresarial (CCE), se construirá un índice de la Función de Auditoria (Index_FA), similar al propuesto por Jiménez, Uribe, Arteaga, \& Moreno (2014), cuya expresión es la siguiente:

Donde:

$$
\text { Index_FA }=\sum \mathrm{R}(\mathrm{Si}) / \mathrm{RP}(\mathrm{Si})+\mathrm{RP}(\mathrm{No})
$$

IAERS = Índice de la función de auditoría

$\mathrm{Rsi}=$ Respuestas con valor de 1 o respuesta "si"

$\mathrm{Rno}=$ Respuestas con valor de 0 o respuesta "no"

Los resultados de los análisis descriptivos del índice de la Función de Auditoría por año, se presentan en la Figura 1, donde se puede observar al año 2013, con el mayor cumplimiento de las disposiciones emitidas por la Comisión Nacional Bancaria y de Valores (CNBV), referentes a la Función de Auditoría. Sin embargo, existe una tendencia negativa en el cumplimiento para el año 2014. 
Figura 1: Comportamiento del Indice de la Funcion de Auditoría, por año

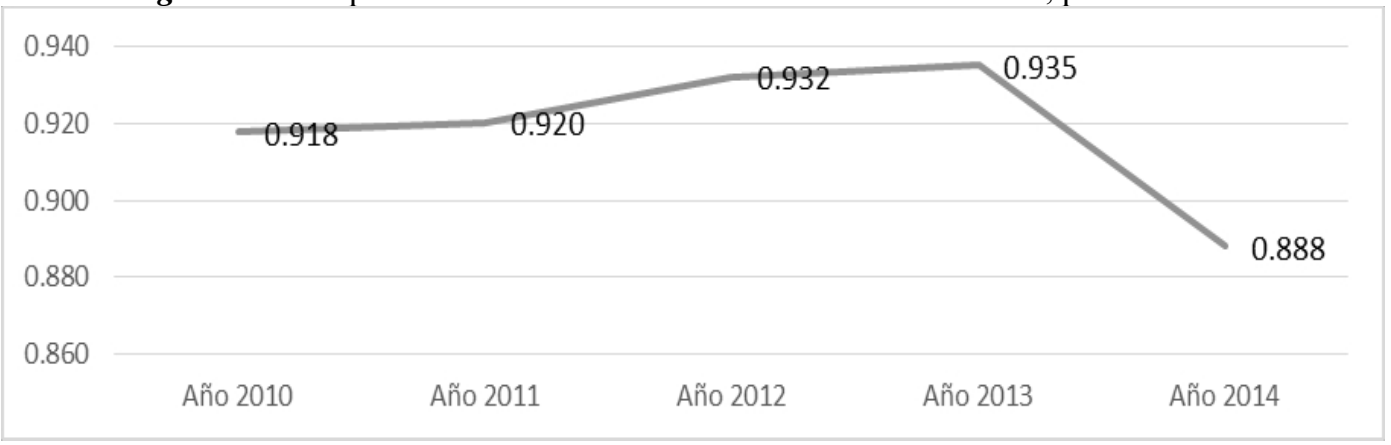

Fuente: Elaboracion Propia

Respecto al sector de pertenencia, las empresas que mayor cumplimiento presentan a la Función de Auditoría, son las dedicadas al sector materiales, con un $95.50 \%$ de adhesión. Por el contrario, las compañías pertenecientes al sector industrial, presentaron el cumplimiento más bajo, con apenas el $88.70 \%$, como lo muestra la Figura 2.

Figura 2: Comportamiento del Índice de la Función de Auditoría por sector de pertenencia

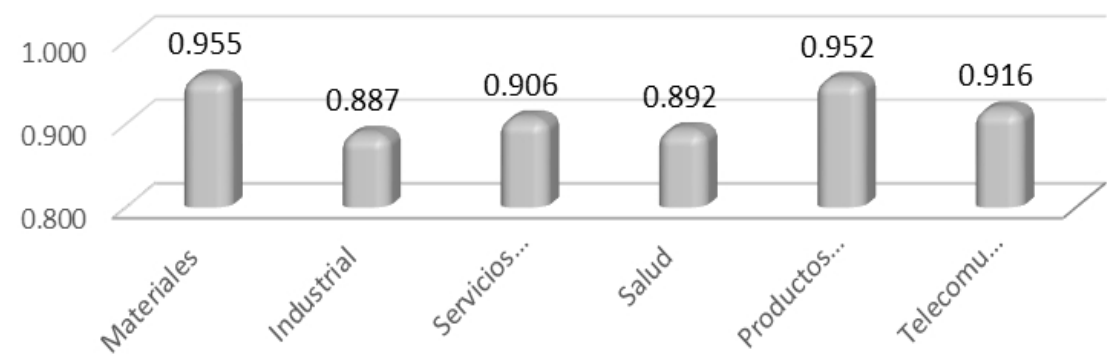

Fuente: Elaboración Propia

Por región geográfica, la Figura 3, nos muestra que las empresas ubicadas en el sur del país, cumplieron con un mayor número de recomendaciones relativas a la Función de Auditoria, con un $96.20 \%$ de ellas. Por el contrario, las compañías que se ubican en el centro del país, solo alcanzaron el $91.10 \%$ 
Figura 3: Análisis del Índice de la Función de Auditoría por región geografica

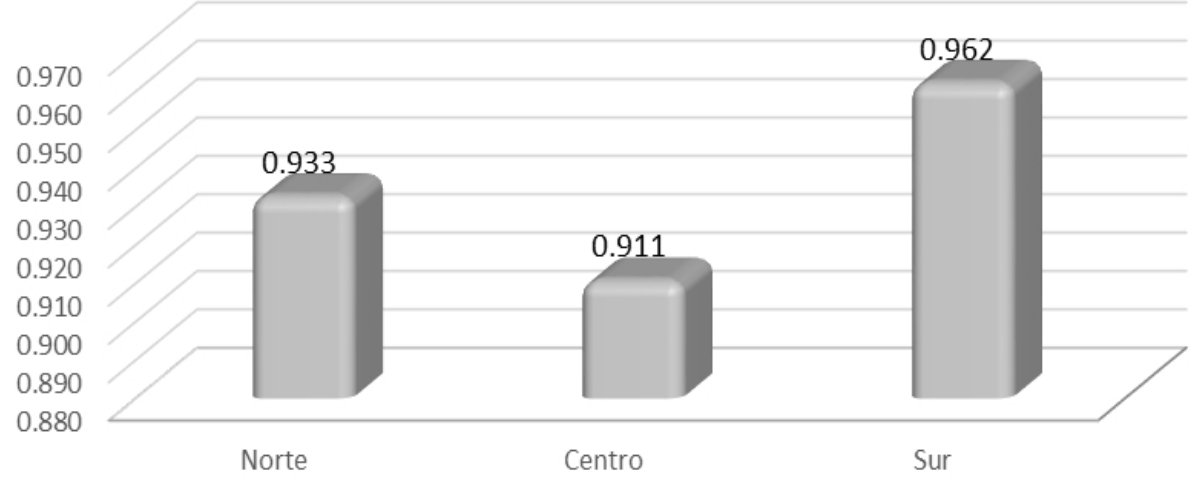

Fuente: Elaboracion Propia

Respecto de la rentabilidad, la Figura 4, nos muestra que las empresas con una rentabilidad media alcanzan un $92.90 \%$ de cumplimiento, y son las empresas que se adhieren en mayor medida a lo dispuesto por la CNBV, en lo referente a las actividades de Auditoría.

Figura 4: Análisis del Índice de la Función de Auditoría en función de la rentabilidad

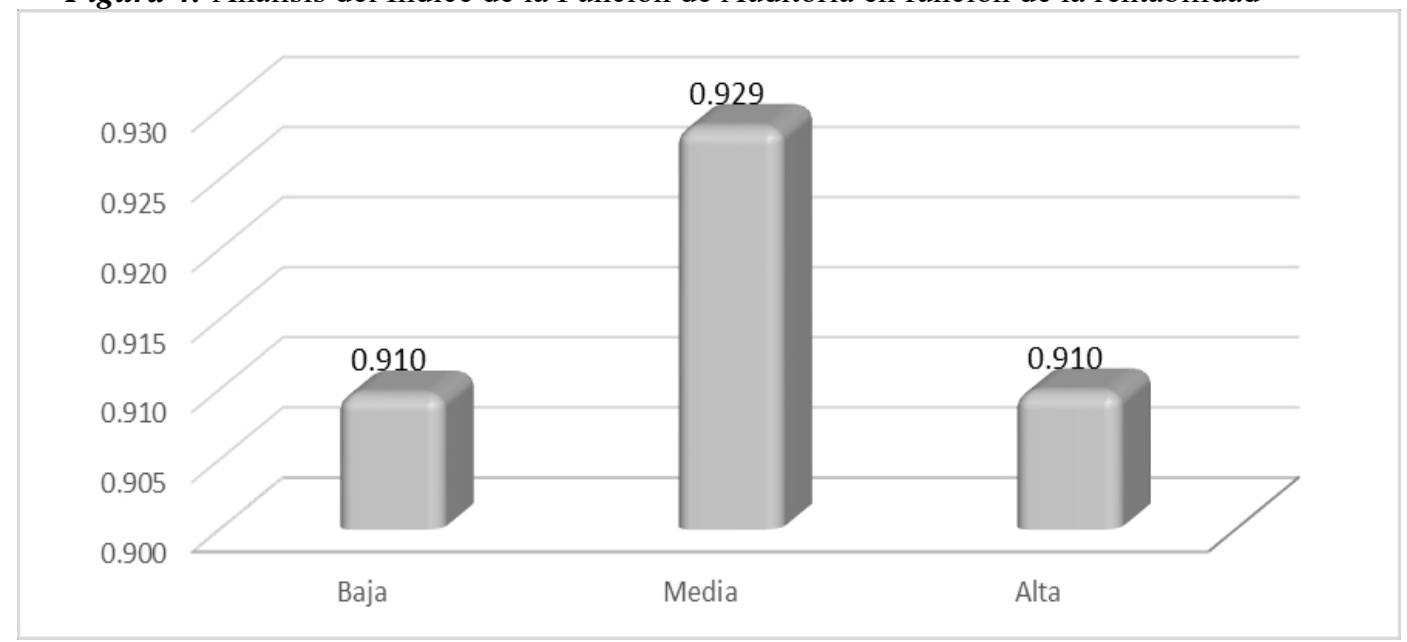

Fuente: Elaboracion Propia

Por tamaño, las compañías grandes (medido por el valor de sus activos al 31 de diciembre de cada año), presentan el mayor cumplimiento del Código de Prácticas Corporativas con un $92.20 \%$, seguidas de las calificadas medianas con un $92.10 \%$ (Figura 5) 
Figura 5: Análisis del Índice de la Función de Auditoría por tamaño

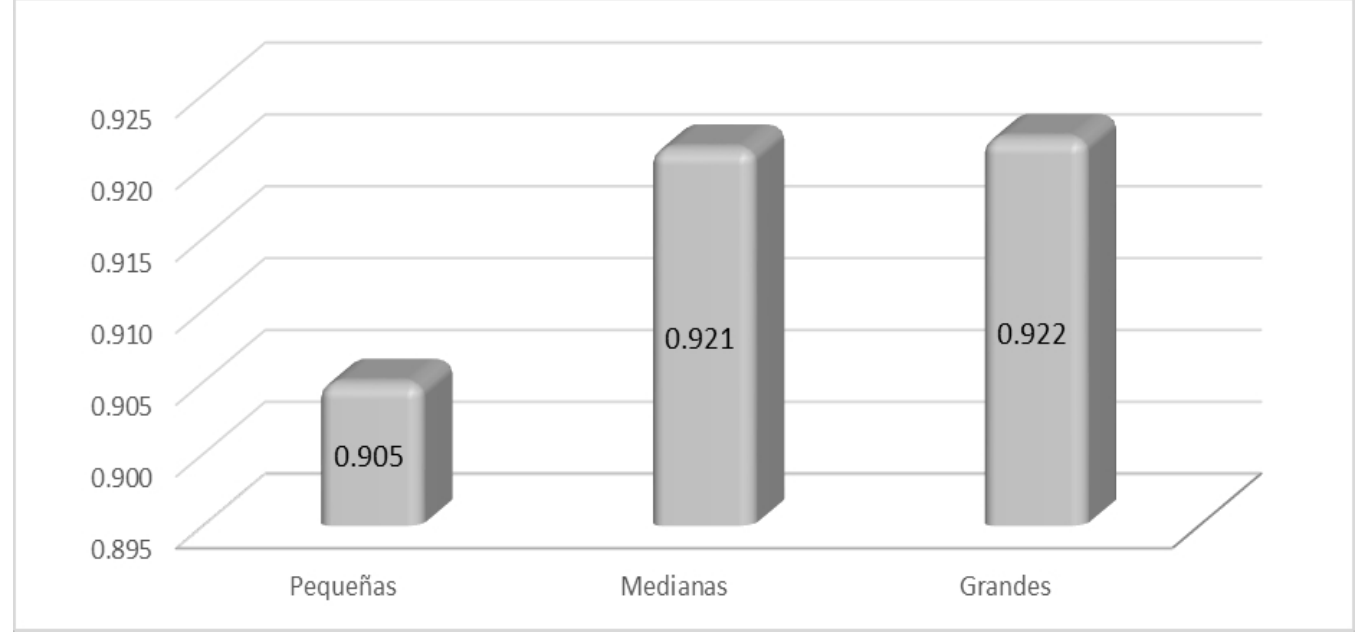

Fuente: Elaboracion Propia

\section{ANÁLISIS MULTIVARIANTE O (PRUEBAS PARAMÉTRICAS) TÉCNICAS DE ANÁLISIS}

Con el propósito de dar a conocer la relación que guarda el índice de la Función de Auditoría, con determinadas características empresariales como son, la región, el tamaño, el sector de pertenencia, el año del informe y el performance organizacional (rentabilidad financiera); y una vez corroborado, que los datos persiguen una distribución normal de frecuencia (Test de Kulmogorov Smirnoff), a través del paquete estadístico SPSS (Paquet Stadistic Social Science versión 15), se analiza la información y se contrastan las hipótesis por medio de pruebas paramétricas (modelos de regresión), cuya expresión es la siguiente:

$$
R F_{i}=\beta_{0}+\beta_{1} S e+\beta_{2} A+\beta_{3} R p+\beta_{4} T+\beta_{5} I N D E X X_{-} F A+\beta_{6} P 1+\varepsilon
$$

Donde:

$\mathrm{RF}=$ Rentabilidad Financiera

$\mathrm{Se}=$ Sector de pertenencia de las empresas

$\mathrm{A}=$ Año del informe

$\mathrm{Rp}=$ Región Geográfica

INDEX_FA= Índice de la Función de Auditoría

Para evitar problemas de heterocedasticidad y multicolinealidad, se aplica el suplemento del Statistical Package for the Social Sciences (SPSS) versión 15 "Determinación de los coeficientes por métodos robustos de la varianza", que nos permite cumplir con los supuestos clásicos de linealidad de los modelos de regresión.

La Tabla 3, muestra los resultados del modelo de regresión lineal, que presentan una relación estadísticamente significativa entre las variables Índice de la Función de Auditoría (INDEX_FA) y el Tamaño de la empresa (medido 
por el total de activos al 31 de diciembre de cada año) de tipo positivo, lo que significa en el primer caso, que el cumplimiento a las disposiciones contenidas en el CMPC emitido por la Comisión Nacional Bancaria y de Valores, relativas a la Función de Auditoria, aumenta la rentabilidad financiera de la empresas de la muestra, los resultados ponen de manifiesto el acierto de los organismos reguladores mexicanos al crear medidas, que apoyen a las organizaciones listadas en su mercado financiero. De esta manera, se acepta la $H_{1}$ : El órgano intermedio que desempeña la función de auditoria influye significativamente en la rentabilidad de la organización.

Respecto a la variable Tamaño, la relación significativa positiva muestra que a mayor tamaño empresarial, mayor será la rentabilidad financiera de las compañías de la muestra, aceptamos la hipótesis $\mathrm{H}_{5}$ : $\mathrm{El}$ tamaño de las empresas, es un factor de influencia de la rentabilidad financiera.

Por el contrario, se rechazan las hipótesis 2, 3 y 4 ya que no presentan significatividad en el modelo de contraste.

Tabla 3: Modelo de Regresión Lineal

\begin{tabular}{|l|l|l|l|}
\hline \multicolumn{2}{|c|}{$R F_{i}=\beta_{0}+\beta_{1} S e+\beta_{2} A+\beta_{3} R p+\beta_{4} T+\beta_{5} I N D E X F A+\beta_{6} P 1+\varepsilon$} \\
\hline \multicolumn{1}{|c|}{ Variable } & \multicolumn{1}{c|}{ Coeficiente } & \multicolumn{1}{c|}{ Std. Error } & \multicolumn{1}{c|}{ Sig. } \\
\hline (Constante) & 202.124 & 158.892 & 0.204 \\
\hline Sector de pertenencia & 0.084 & 0.073 & 0.251 \\
\hline Año de informe anual & -0.102 & 0.079 & 0.198 \\
\hline $\begin{array}{l}\text { Región del país en la que se encuentra } \\
\text { la empresa }\end{array}$ & -0.158 & 0.269 & 0.558 \\
\hline Tamaño & 0.227 & 0.14 & $0.107 *$ \\
\hline INDEX_FA & 2.009 & 1.062 & $0.059^{* *}$ \\
\hline \multicolumn{3}{|c|}{0.33} \\
\hline R-Cuadrado & 0.000 \\
\hline ANOVA & \multicolumn{3}{c}{ Significativo al 99\%, **Significativo al 95\%, *Significativo al 90\% } \\
Variable dependiente: Rentabilidad Financiera
\end{tabular}

\section{Conclusion}

El propósito de la presente investigación, fue demostrar a través de estudios empíricos el nivel de cumplimiento y la influencia que mantienen los mecanismos del Gobierno Corporativo, relativos a la Función de Auditoria en la rentabilidad financiera de una muestra de empresas listadas en la Bolsa Mexicana de Valores en el periodo de 2010 al 2014. Los resultados descriptivos del Índice de la Función de Auditoria (INDEX_FA), muestran que son numerosas las compañías que aún no cumplen con las disposiciones de la CNBV al $100 \%$.

El análisis de las tendencias por año, presentan comportamientos negativos al final del estudio, lo que significa, que las compañías lejos de alcanzar el cumplimiento total, se alejan de él. 
Por otro lado, los resultados del modelo de regresión lineal, muestra una relación significativa entre el ratio Rentabilidad Financiera, el INDEX_FA y el tamaño empresarial de tipo positivo, relación que nos puede permitir suponer, que el apego al índice, aumenta la rentabilidad de las organizaciones y por tanto, más atractivas serán para el público inversionista. En cuanto al tamaño, los resultados nos permiten observar, que se trata de una variable, que influye en la obtención de resultados financieros favorables para las compañías analizadas. Por último, se deberían endurecer las medidas para que las empresas listadas, se adhieran a los mecanismos de Gobierno Corporativo al $100 \%$.

En definitiva, las disposiciones emitidas por la Comisión Nacional Bancaria y de Valores a través del CMPC, aumentan la rentabilidad de las organizaciones y las convierten más atractivas en el mercado bursátil.

\section{References:}

1. Abbadi, S., Hijazi, Q., \& Rahahleh, A. (2016). "Corporate Governance Quality and Earnings Management: Evidence from Jordan". Australasian Accounting, Business and Finance Journal, $10(2), 52-75$.

2. Acosta, D., Barreda, P., Diaz, I., \& Visso, D. (26 de Julio de 2016). Repositorio Académico Universidad Peruana de Ciencias Aplicadas. Obtenido de Repositorio Académico Universidad Peruana de Ciencias Aplicadas:

http://repositorioacademico.upc.edu.pe/upc/handle/10757/618351

3. Benavides, J., \& Mongrut, S. (2010). "Códigos del gobierno: hecho o ficción un estudio de los códigos del gobierno en Colombia”. Estudios Gerenciales Universidad ICESI, 85-118.

4. Bota, C. (2013). "Linking governance to efficacy of corporate boards: a global perspective”. Procedia Social and Behavioral Sciences, 99, 1073-1081.

5. Chen, S., \& Chen, I. (2012). “Gobierno corporativo y la asignación de capital de diversas empresas". Journal of Banking and Finance, 15, 273-289.

6. Chris, G., Theodoros, K., \& Vasilios, C. (2014)."Corporate governance in practice. The Greek case”. Economics and Finance, 9, 369-379.

7. Cueto, D. (2013). "La Sustituibilidad y la Complemetariedad de los mecanismos de Gobierno Corporativo en América Latina”. International Review of Economics and Finance, 25.

8. Feras, M. (2013). "The impact of Corporate Governance on the Financial Outcomes of Global Diversification”. The International Journal of Accounting, 48, 364-389. 
9. Javaid, F., \& Saboor, A. (2015). "Impact of Corporate Governance index on Firm Performance: evidence from Pakistani manufacturing sector". Journal of Public Administration and Governance, 5(2).

10. Jiménez, S., Gallardo, R., \& Alvarado, M. (2-4 de Octubre de 2013). Congreso Internacional de Contaduría, Administración e Informática. Recuperado el 25 de 07 de 2017, de http://congreso.investiga.fca.unam.mx/docs/xviii/docs/5.06.pdf

11. Jiménez, S., Uribe, H., Arteaga, G., \& Moreno, E. (2014). “Análisis del cumplimiento del código de mejores prácticas corporativas en México”. Boletín Científico de las Ciencias Económico Administrativas del ICEA, 3(5).

12. Jiraporn, P., \& Kim, J. (2012). "Capital structure and corporate governance quality: Evidence from the Institutional Shareholder Services". International Rewiew of Economics and Finance, 22, 208221.

13. Lagos. (2014). "Influencia del gobierno corporativo en el costo de capital". Revista Estudios gerenciales, 30, 73-84.

14. Lakshan, \& Wjekoon. (2012). "El gobierno corporativo y el fracaso empresarial. Procedia Economics and Finance", 2, 191-198.

15. Latief, R., Hassan, S., \& Ahmed, S. (2014). "Impact of Corporate Governance on Performance of Privatized Firms; Evidence from NonFinancial Sector of Pakistan". Middle-East Journal of Scientific Research, 19(3), 360-366.

16. Lim, M. (2013). "Corporate ownership, corporate governance reform and timeliness of earnings: Malaysian evidence". Journal of Contemporary Accounting and Economics (10), 32-45.

17. Martínez , L. (2011). "El Papel de los Consejos de Administración en el Gobierno Corporativo para el caso de México”. Tecnológico de Monterrey, 29-37.

18. Prommin, P., Jumreornvong, S., \& Jiraporn, P. (2014). "The effect of corporate governance on stock liquidity: The case of Thailand". International Review of Economics and Finance, 32, 132-142.

19. Puspitaninggrum. (2012). "Corporate governance mechanism and the level of internet financial reporting: Evidence from Indonesian companies". Procedia Economics and Finance, 2, 157-166.

20. Stefanescu, C. (2011). "Disclosure and transparency in corporate governance codes -comparative analysis with prior literature findings". Procedia Economics and Finance, 113-114. 